\title{
Clinical Characteristics of Febrile UTI First Developed Over 5 Years of Age
}

\section{Da Eun Roh, M.D. Hyo Rim Suh, M.D. So Yoon Min, M.D. Tae Kyoung Jo, M.D. Hee Sun Baek, M.D. Min Hyun Cho, M.D.}

Department of Pediatrics, Kyungpook National University, School of Medicine, Daegu, Korea

\author{
Corresponding author: \\ Min Hyun Cho, M.D. \\ Department of Pediatrics, Kyungpook \\ National University Medical Center \\ 807 Hogukno, Buk-gu, Daegu, 41404 \\ Korea \\ Tel: +82-53-200-2741 \\ Fax: +82-53-200-2039 \\ E-mail:chomh@knu.ac.kr
}

Received: 13 February 2017

Revised: 15 March 2017

Accepted: 4 April 2017
Purpose: Febrile urinary tract infection (UTI) is one of the commonest bacterial infections in children. The purpose of this study is to investigate the clinical characteristics of the first episode of febrile UTI occurring in children over 5 years compared to those in infants younger than a year.

Methods: We retrospectively reviewed the medical records of 10 patients over 5 years, having febrile UTI, and 25 controls under 1 year. Clinical characteristics including symptoms at admission, the time interval between symptom onset and hospital visit and/or diagnosis, duration of fever, urinalysis, and other laboratory and imaging test results were compared between the two groups.

Results: Most patients in the control group showed only high fever at the time of presentation to the hospital. However, $60 \%$ of the case group had fever along with gastrointestinal (Gl) symptoms such as abdominal and flank pain, vomiting, as well as relatively mild pyuria. The case group showed a longer duration between symptom onset and hospital visit and/or diagnosis.

Conclusions: Delay in diagnosis and initiation of treatment of UTI increases the risk of permanent renal scarring and associated complications. Therefore, early diagnosis and treatment of febrile UTI is vital for very young infants, as well as children considering that febrile UTI could be an important cause of febrile illness in children over 5 years.

Key words: Urinary tract infection, Children, Age

\section{Introduction}

Febrile urinary tract infection (UTI) is one of the most common bacterial illnesses in children. As febrile UTI may lead to acute renal parenchymal damage, possible permanent renal scarring, decreased renal function and endstage renal disease, early diagnosis and treatment are important. The majority (over 70-80\%) of febrile UTI during childhood is known to occur in the first year of life with a male predominance ${ }^{1,2)}$. Infants are more likely to have febrile UTI than elderly due to immune system immaturity, high incidence of systemic infections, and anatomical anomalies of urinary tract ${ }^{3}$. One of the reasons of male predominance in infants is high incidence of urinary tract anomalies such as vesicoureteral reflux (VUR ${ }^{4)}$.

Children with UTI present various symptoms and signs depending on age. Generally, infants under 1 year of age have non-specific symptoms such as 
fever, vomiting, lethargy, poor oral intake, and diarrhea. Contrastively, children older than 5 years are known to have specific and localized symptoms and signs associated with UTI such as tenderness on costovertebral angle, urinary frequency, dysuria, and urinary urgency, as well as fever. As the prevalence of UTI in infants under 1 year of age is high, but they usually present non-specific clinical symptoms, most of pediatricians preferentially perform urinalysis to rule out UTI in infants with fever ${ }^{5)}$. Additionally, for this reason, there have been various reports on the clinical symptoms and early diagnosis of UTI in infants under 1 year of age ${ }^{6}$. However, in the cases of older children, sometimes, the first episode of febrile UTI is difficult to diagnose. Especially, if they present gastrointestinal symptoms such as abdominal pain or vomiting with fever, many physicians tend to underestimate the possibility of febrile UTI in comparison to the cases of infants. As a result, performing urinalysis may be delayed.

This study was performed to investigate the clinical manifestations of first UTI developed from children over 5 years of age and apply proper diagnosis and therapeutic process of the first episode of febrile UTI according to the age difference.

\section{Material and methods}

We reviewed the medical records of 10 patients with the first episode of febrile UTI developed over 5 years of age and 25 patients under 1 year of age as controls, the common age for febrile UTI, retrospectively. The patients were admitted to Kyungpook National University Children's hospital between January 2013 and June 2016.

Clinical characteristics included main symptoms at the admission, the time from symptom onset to the hospital visit and diagnosis, the duration of fever, urinalysis and imaging test. The degree of pyuria was classified as mild and severe pyuria according to the number of WBC in the urinalysis. Severe pyuria was defined as showing a WBC count of 20 or more. In contrast, mild pyuria was defined as showing a WBC count less than 20. UTI diagnosis was based on fever (axillary temperature $\geq 38^{\circ} \mathrm{C}$ ) and positive urine culture, defined by a single microorganism present no less than $10^{5}$ colony-forming unit (CFU)/mL in a spe- cimen obtained via catheterization, bag urine or clean voided midstream urine or no less than $10^{3} \mathrm{CFU} / \mathrm{mL}$ in suprapubic collection. Moreover, febrile UTI was considered if there are positive findings of acute pyelonephritis (APN) in renal ultrasonography or abdominal CT even though they showed negative urine culture results. Patients with previous histories of UTI were excluded.

Renal ultrasonography (USG) and technetium-99mlabeled dimercaptosuccinic acid (DMSA) scintigraphy were performed in all patients of control group. Voiding cystourethrography (VCUG) was performed on patients of control group who showed a renal parenchymal lesion in DMSA renal scintigraphy. In case group, renal USG or abdominal CT was performed selectively according to the clinical severity and the usefulness of differential diagnosis. DMSA renal scintigraphy was performed in all patients of case group. VCUG was selectively performed on the patients of case group with positive DMSA renal scintigraphy results which showed renal parenchymal damage, considering clinical features, age, and ease of test.

The criteria for renal abnormality in renal USG were as follows; increase in renal size, presence of hydronephrosis, focal or diffuse change of parenchymal echogenicity (hyper-echogenicity or hypo-echogenicity) and loss of corticomedullary differentiation. The criteria for renal abnormality in abdominal CT were as follows; attenuation change due to edema or hemorrhage, renal abscess, renal size change and urinary tract obstruction. The abnormal findings in DMSA renal scintigraphy were as follows: focal or diffuse areas of decreased uptake of labeled DMSA or diffusely decreased uptake in an enlarged kidney. The degree of vesicoureteral reflux was classified as I, II, III, IV, and V according to the international reflux study committee classification.

All results were analyzed with t-test for continuous variables and chi-square test for categorical variables using SPSS (Version 10.0). A $P$-value of less than 0.05 was considered statistically significant.

\section{Results}

The mean age of the control group was $0.4 \pm 0.2$ years. In patients under 1 year of age, the prevalence of febrile UTI 
was higher in males (n: 18, 72\%) than in females (n: 7, 28\%). The mean age of the case group was $9.4 \pm 3.7$ years. In patients over 5 years of age, prevalence of first episode of febrile UTI was significantly higher in female (n: 9, 90\%) than males (n: 1, 10\%) (Table 1).

All the patients in control group showed only symptom of high fever at the time of hospital visit. In comparison, $60 \%$ of the case group showed gastrointestinal symptoms such as abdominal pain, flank pain and vomiting as well as fever. In case group, 3 of the patients showed urinary symptoms such as urinary frequency, dysuria, urgency,

Table 1. Baseline Characteristics of the Patients

\begin{tabular}{|c|c|c|c|}
\hline & $\begin{array}{l}\text { Control } \\
\text { group } \\
(n=25)\end{array}$ & $\begin{array}{l}\text { Case } \\
\text { group } \\
(n=10)\end{array}$ & $\begin{array}{c}P_{-} \\
\text {value }\end{array}$ \\
\hline Age (years) & $0.4 \pm 0.2$ & $9.4 \pm 3.7$ & \\
\hline Sex (male/female) & $18 / 7$ & $1 / 9$ & \\
\hline \multicolumn{4}{|l|}{ Symptom at diagnosis } \\
\hline Fever (axillary temperature $\geq 38^{\circ} \mathrm{C}$ ) & $25 / 25$ & $10 / 10$ & \\
\hline Abdominal pain & $0 / 25$ & $2 / 10$ & $<0.05$ \\
\hline Flank pain & $0 / 25$ & $3 / 10$ & $<0.05$ \\
\hline Vomiting & $0 / 25$ & $3 / 10$ & $<0.05$ \\
\hline $\begin{array}{l}\text { Urinary symptoms (frequency/ } \\
\text { dysuria/urgency/hematuria) }\end{array}$ & $0 / 25$ & $3 / 10$ & \\
\hline \multicolumn{4}{|l|}{ Degree of pyuria in urinalysis } \\
\hline$W B C>20 / H P F$ & $20 / 25(80 \%)$ & $6 / 10(60 \%)$ & \\
\hline$W B C<20 / H P F$ & $5 / 25(20 \%)$ & $4 / 10(40 \%)$ & \\
\hline
\end{tabular}

Abbreviations: WBC, white blood cell count; HPF, high-power field. Values: Mean \pm Standard deviation. hematuria at the time of diagnosis (Table 1). In case group, one of the patients showed nocturnal enuresis and two of the patients showed constipation as underlying disease or accompanying symptoms before febrile illness. However, there were no other urinary symptoms such as urinary frequency or urinary incontinence which associated with bladder function before the onset of febrile illness. In $80 \%$ of patients in control group, severe pyuria with WBC> 20/ HPF was observed. On the other hand, only $40 \%$ of patients in case group showed pyuria with WBC $>20 / \mathrm{HPF}$ and $40 \%$ of patients in case group showed mild pyuria with $<20 /$ HPF (Table 1).

The mean duration from symptom onset to hospital visit (2.4 \pm 1.3 vs $1.6 \pm 0.9$ days, $P<0.05$ ), from symptom onset to final diagnosis ( $2.8 \pm 1.6$ vs $1.6 \pm 0.9$ days, $P<0.05$ ), and of fever lasting ( $2.0 \pm 0.9$ vs $1.3 \pm 0.5$ days, $P<0.05$ ) was significantly longer in case group than control group (Table 2 and Fig. 1).

One of the control group patients showed urinary tract anomalies on abdominal CT. No urinary tract anomalies

\begin{tabular}{|c|c|c|c|}
\hline Mean time(days) & Control group & Case group & $P$-value \\
\hline $\begin{array}{l}\text { A. To visit hospital after } \\
\text { symptom onset }\end{array}$ & $1.6 \pm 0.9$ & $2.4 \pm 1.3$ & $<0.05$ \\
\hline B. Duration of fever & $1.3 \pm 0.5$ & $2.0 \pm 0.9$ & $<0.05$ \\
\hline $\begin{array}{l}\text { C. To final diagnosis after } \\
\text { symptom onset }\end{array}$ & $1.6 \pm 0.9$ & $2.8 \pm 1.6$ & $<0.05$ \\
\hline
\end{tabular}
symptom onset

Values: Mean \pm Standard deviation.

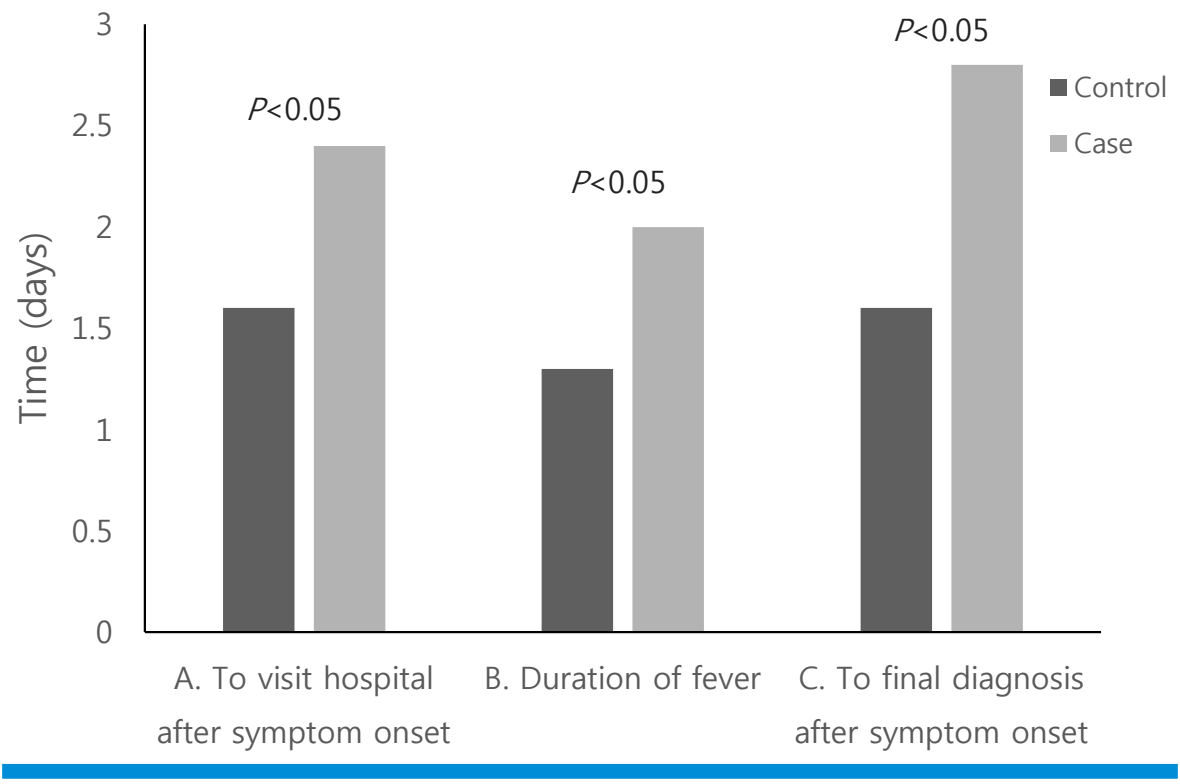

Fig. 1. Comparison of clinical manifestations of patients between age groups. 
were found in the case group, and there was no statistical significance between two groups. DMSA renal scintigraphy was performed on all patients with confirmed UTI. 9 of control group subjects (36\%) and 7 of case group (70\%) showed abnormal DMSA scintigraphy results, respectively. The incidence of renal parenchymal damage was relatively higher in the case group, but there was no statistical significance. VCUG was performed in 12 of 25 control subjects, and VUR was found in 2 of them. And VCUG was performed in 4 of 10 case subjects, and VUR was found in 2 of them. The prevalence of VUR was relatively higher in the case group, but there was no statistical significance (Table 3).

\section{Discussion}

The prevalence of UTI in children under 2 years presenting fever has been the subject of several large prospective study and a meta-analysis ${ }^{4,78}$. The overall prevalence of UTI is approximately 7 percent in febrile infants and young children. Among febrile infants less than 1 year of age, the prevalence of febrile UTI in male and female was $27.5 \%$ and $21.5 \%$, respectively. In the pooled analysis of four studies that included children under 19 years -most of them were older than 2 years- and had urinary symptoms and/or fever, the prevalence of UTI was 7.8 percent. In elderly children who were toilet-trained, the majority of febrile UTI developed in female, and clinical symptoms were different depending on their age. Generally, infants under 1 year of age have non-specific symptoms such as fever, vomiting, lethargy, poor oral intake, and diarrhea. Contrastively, children older than 5 years are known to have specific and localized symptoms and signs associated with UTI such as tenderness on costovertebral angle, urinary frequency, dysuria, and urinary urgency, as well as fever ${ }^{9,10)}$. However, febrile UTI was difficult to diagnose due to non-specific symptoms in elderly children as well as infants.

In this study, although most of patients in control group showed only high fever at the time of hospital visit, diagnosis of UTI was done promptly because many physicians have preferentially considered the possibility of UTI as the common reason of fever in infants. In comparison, $60 \%$ of the case group showed GI symptoms as well as fever, so urinalysis tended to be delayed and early diagnosis was often difficult because it was likely to be confused with other febrile diseases.

Comparing urinalysis at the time of diagnosis, $80 \%$ of infants under 1 year of age showed severe pyuria with WBC $>20 / \mathrm{HPF}$, while $40 \%$ of children after 5 year of age had relatively mild pyuria with $\mathrm{WBC}<20 / \mathrm{HPF}$. In this study, milder pyuria in case group than control group might be related to the severity of dehydration due to the different amount of fluid intake or the previous use of antibiotics before diagnosis. In this study, many of children over 5 years of age had a higher use of antibiotics prior to diagnosis of UTI because they often visited hospitals after using empirical antibiotics for other infections due to non-specific symptoms and lower possibility of UTI.

In case group, the mean duration to visit the hospital after symptom onset was significantly longer than that of infants under 1 year of age. This may be related to the time spent on empirical antibiotics use or conservative treatment in primary care center before diagnosis of UTI due to non-specific symptoms. The difference in hospital visit

Table 3. Imaging Test Results

\begin{tabular}{|c|c|c|c|c|}
\hline & Negative & Positive & Total & Ratio of positive results (\%) \\
\hline \multicolumn{5}{|c|}{ Anomalies of urinary tract on renal USG or Abdominal CT } \\
\hline Control group & 24 & 1 & 25 & 4 \\
\hline Case group & 10 & 0 & 10 & 0 \\
\hline \multicolumn{5}{|c|}{ DMSA renal scintigraphy } \\
\hline Control group & 16 & 9 & 25 & 36 \\
\hline Case group & 3 & 7 & 10 & 70 \\
\hline \multicolumn{5}{|l|}{ VCUG } \\
\hline Control group & 10 & 2 & 12 & 17 \\
\hline Case group & 3 & 2 & 5 & 40 \\
\hline
\end{tabular}

Abbreviations: USG, ultrasonography; CT, computed tomography; DMSA, dimercaptosuccinic acid;VCUG, voiding cystourethrography. 
rates or admission criteria between infants and elderly children can also be considered as possible causes.

The mean duration to final diagnosis after symptom onset and fever duration after visiting hospital was significantly longer in the case group. This means that it takes a longer time to visit the hospital and accurate diagnosis and proper treatment are delayed in case group. In previous studies, fever duration, peak fever and therapeutic delay time have been reported to be important predictive factors of acute photon defect and renal scar formation in DMSA renal scintigraphy ${ }^{11-15)}$. Acute inflammatory reactions, such as toxic metabolite release from infiltrating polymorphonuclear leukocytes, have been observed to damage the renal tissue, and the duration of the reaction may determine the severity and extent of the lesions ${ }^{16}$. The degree of acute inflammatory reaction prior to initiation of proper treatment is associated with renal tissue damage and ultimate scar formation ${ }^{11)}$. However, most studies on the association of early diagnosis and therapeutic delay with renal scar formation focused on infants under 1 year of age. Research on elderly children is relatively inadequate.

DMSA renal scintigraphy is the most widely used sensitive test for the detection of renal parenchymal damage in febrile UTI. Previous studies have reported that duration of fever, blood leukocyte count and higher C-reactive protein level were correlated with the development renal scars ${ }^{11)}$. Previous study reported that females and children older than 1 year presented with significantly higher rates of abnormal DMSA scintigraphy result and VUR presented by VCUG $^{2,15,17)}$.

The correlation between age and renal scar formation has not been consistently reported among studies. In previous studies, younger infants have been reported to be more susceptible to renal scar formation because of weak resistance to infection. However, recent studies of DMSA scintigraphy follow-up reported that children over 1 year of age are more likely to develop renal scar after initial $\mathrm{UTI}^{17,18)}$. Benador et al. reported that renal scar formation was more common in children above 1 year of age than those below this age ${ }^{13)}$. Kim et al. reported a significantly high positive rate of abnormal DMSA findings in older children who were clinically suspected for APN, particularly older than 1 year of age ${ }^{2}$. This is thought to be due to the progressive renal damage from reinfection and previous unrecognized
UTI without treatment. In our study, the relative high incidence of acute cortical defects in elderly children is similar to the results of previous studies even though there were no statistically significant differences.

Renal parenchymal damage caused by febrile UTI may be reversible and all renal damages do not ultimately lead to scar. However, delay of diagnosis and treatment initiation of UTI increases permanent renal scarring and associated complications. Therefore, early diagnosis and treatment initiation of febrile UTI are important for all ages including infant and elderly children. As infants under 1 year of age show ambiguous symptoms and difficulties to diagnose, there have been many studies about the necessity of early diagnosis and treatment at this age. However, in this study, we retrospectively investigated cases of febrile UTI first diagnosed in the children over 5 year of age and we have identified the clinical characteristics at this age: 1) Early diagnosis is difficult to make because most of the patients had fever and other nonspecific symptoms 2) Diagnosis becomes more ambiguous with the use of empirical antibiotics because of misdiagnosis of other infectious disease in primary care 3) Initiation of treatment is delayed 4) For this reason, risk of renal damage in the DMSA scintigraphy is relatively high. Additionally, even if clinical features are not severe, abnormal findings are often seen on imaging such as abdominal CT, DMSA scintigraphy, VCUG. For this reason, imaging evaluation should be actively performed in elderly children with febrile UTI.

This study has some limitations. First, this study was a single-center based, so the number of registered patients was relatively small. Second, there was the possibility of selection bias because control group was partially composed of infants with clinical course of typical UTI. Third, it was hard to do invasive procedure such like VCUG to older children such as adolescent girl.

Febrile UTI may cause significant febrile illness over 5 year of age, so attention and concern of parents and pediatricians are demanded.

\section{Conflicts of interest}

No potential conflict of interest relevant to this article was reported. 


\section{References}

1. Lee KY. New insights for febrile urinary tract infection (acute pyelonephritis) inchildren. ChildKidney Dis 2016;20:37-44.

2. Kim JH, Kim MJ, Choi BM, Yoo KH, Hong YS, Lee JW. The diagnostic value of clinical and radiologic findings in children after the first episode of acute pyelonephritis. J Korean Soc Pediatr Nephrol 2005;9:201-12.

3. Coe HJ, Kim TH, Cho H, Kim JH. Comparison of the clinical and radiologic characteristics between different age groups with first febrile UTI under 2 years of age. J Korean Soc Pediatr Nephrol 2007;11:229-38.

4. Shaikh N, Morone NE, Bost JE, Farrell MH. Prevalence of urinary tract infection in childhood: a meta-analysis. Pediatr Infect Dis J 2008;27:302-8.

5. Matthews KE, Mines RD, Pakula LC. Practice parameter: the diagnosis, treatment, and evaluation of the initial urinary tract infection in febrile infants and young children. Pediatrics 1999;103:843.

6. Doganis D, Siafas K, Mavrikou M, Issaris G, Martirosova A, Perperidis $G$. Does early treatment of urinary tract infection prevent renal damage? Pediatrics 2007;120:e922-8.

7. Shaw KN, Gorelick M, McGowan KL, Yakscoe NM, Schwartz JS. Prevalence of urinary tract infection in febrile young children in the emergency department. Pediatrics 1998;102:e16.

8. Hoberman A, Chao HP, Keller DM, Hickey R, Davis HW, Ellis D. Prevalence of urinary tract infection in febrile infants. J Pediatr 1993;123:17-23.
9. Lee SJ. Guidelines for childhood urinary tract infection. Korean J Pediatr 2009:52:976-83.

10. Shaikh N, Morone NE, Lopez J, Chianese J, Sangvai S, D'Amico F. Does this child have a urinary tract infection? JAMA 2007;298: 2895-904.

11. Oh MM, Cheon J, Kang SH, Park HS, Lee JG, Moon DG. Predictive factors for acute renal cortical scintigraphic lesion and ultimate scar formation in children with first febrile urinary tract infection. J Urol 2010;183:1146-50.

12. Lee YJ, Lee JH, Park YS. Risk factors for renal scar formation in infants with first episode of acute pyelonephritis: a prospective clinical study. J Urol 2012;187:1032-6.

13. Benador D, Benador N, Slosman D, Mermilllod B, Girardin E. Are younger children at highest risk of renal sequelae after pyelonephritis? The Lancet 1997;349:17-9.

14. An YK, Cho MH, Kim KS. Which factors related to the renal cortical defects in infants under 3 months of age with urinary tract infections? Child Kidney Dis2016;20:57-62.

15. Park YS. Renal scar formation after urinary tract infection in children. Korean JPediatr 2012;55:367-70.

16. Montini G, Tullus K, Hewitt I. Febrile urinary tract infections in children. N Engl J Med 2011;365:239-50.

17. Lee J, Woo BW, Kim HS. Prognostic factors of renal scarring on follow-up DMSA scan in children with acute pyelonephritis. Child Kidney Dis 2016;20:74-8.

18. Seon YS, Kwon DG, Shin YH, Pai KS. Prognostic factors of renal defects on the initial DMSA scan in children with acute pyelonephritis. J Korean Soc Pediatr Nephrol 2010;14:195-202. 